\title{
An apparent case of undiagnosed donor Kawasaki disease manifesting as coronary artery aneurysm in a pediatric heart transplant recipient
}

Friedland-Little J, Aiyagari R, Gajarski RJ, Schumacher KR. An apparent case of undiagnosed donor Kawasaki disease manifesting as coronary artery aneurysm in a pediatric heart transplant recipient

Abstract: We present a case of coronary ectasia and LAD coronary artery aneurysm with angiographic characteristics of Kawasaki disease in a three-yr-old girl two-yr status post-orthotopic heart transplant. Coronary anomalies were noted during initial screening coronary angiography two yr after transplant. Subsequent review of the donor echocardiogram revealed that the LMCA had been mildly dilated prior to transplant. In the absence of any symptoms consistent with Kawasaki disease in the transplant recipient, this appears to be a case of Kawasaki disease in the organ donor manifesting with coronary anomalies in the transplant recipient. The patient has done well clinically, and repeat coronary angiography has revealed partial regression of coronary anomalies. Given multiple reports in the literature of persistent abnormalities of coronary artery morphology and function after Kawasaki disease, close monitoring is warranted, with consideration of potential coronary protective medical therapies.

\author{
Joshua Friedland-Little, Ranjit \\ Aiyagari, Robert J. Gajarski and Kurt \\ R. Schumacher \\ Division of Pediatric Cardiology, Department of \\ Pediatrics, University of Michigan Health Center, \\ Ann Arbor, MI, USA \\ Key words: heart transplantation - pediatrics - \\ Kawasaki disease - coronary aneurysm \\ Joshua Friedland-Little, MD, University of Michigan \\ Congenital Heart Center, C.S. Mott Children's \\ Hospital, 1540 East Hospital Drive, Ann Arbor, Ml \\ 48109-4204, USA \\ Tel.: +1 7342328431 \\ Fax: +17349369470 \\ E-mail: jmfried@@med.umich.edu \\ Accepted for publication 11 July 2012
}

\section{Background}

First described in 1967 in Japan, Kawasaki disease is an acute, systemic vasculitis of unclear etiology (1). It is the most common cause of acquired heart disease in children in the United States, with coronary artery inflammation and aneurysm formation developing in $15-25 \%$ of untreated patients (2). Standard treatment of Kawasaki disease involves IVIG and high-dose aspirin in the acute phase, with long-term lowdose aspirin therapy in patients with evidence of coronary involvement (2). Coronary artery abnormalities usually develop within six to eight wk of the onset of illness. The coronary artery disease is often reversible, with resolution

\footnotetext{
Abbreviations: AV, atrioventricular; EBV, Epstein-Barr virus; HTx, heart transplantation; IVIG, intravenous immunoglobulin; LAD, left anterior descending; LMCA, left main coronary artery; RCA, right coronary artery; TCAD, transplant coronary artery disease; VA, venoarterial.
}

of coronary artery anomalies seen in approximately $50 \%$ of patients within two yr (3).

\section{Case report}

The patient is a three-yr-old girl with a history of hypoplastic left heart syndrome status posthemi-Fontan procedure who underwent orthotopic HTx at 18 months of age due to poor right ventricular function and significant AV valve regurgitation. Her post-HTx hospital course was complicated by biventricular failure requiring VA ECMO for $36 \mathrm{~h}$, from which she completely recovered. Immunosuppression regimen initially consisted of tacrolimus and mycophenolate mofetil, but the patient was subsequently switched to tacrolimus monotherapy due to persistent EBV viremia. Initial screening coronary angiography performed two yr after her HTx revealed a dilated LMCA along with a fusiform aneurysm of the LAD coronary artery (Fig. 1a). The RCA also demonstrated coronary ectasia with diffuse dilation (Fig. 1b). These angiographic 
findings are characteristics of Kawasaki disease, although the patient did not have any history of febrile illness consistent with the diagnosis. Lack of available medical records has precluded investigating whether the donor, a three-yr-old boy with head trauma, had any history of febrile illness prior to organ donation. Review of the donor echocardiogram, however, revealed that the LMCA had been prominent and mildly dilated prior to transplant (Fig. 2). Review of previous echocardiograms from our institution revealed that the left and right main coronary arteries had been significantly dilated one month after transplant and that the aneurysm of the LAD had been present at that time as well. Repeat angiography one yr later has revealed that the right and left main coronary arteries are now normal in size. The LAD aneurysm is still present, but has become smaller. The patient has continued to do well clinically and is being maintained on low-dose aspirin therapy.

\section{Discussion}

To our knowledge, this is the first reported case in which undiagnosed Kawasaki disease in a pediatric cardiac donor has apparently presented with coronary artery anomalies in the transplant recipient. While it is impossible for us to confirm a diagnosis of Kawasaki disease in the organ donor given the limited available medical records, the presence of coronary ectasia in the donor echocardiogram, as well as the demonstration of coronary anomalies in the transplanted heart as early as a month after transplant,

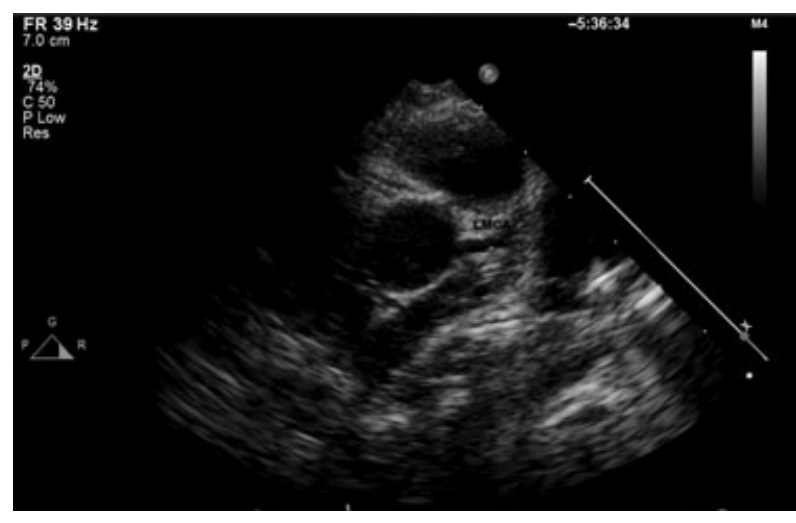

Fig. 2. Donor echocardiogram performed shortly prior to transplant revealed that the LMCA was prominent and mildly dilated at that time, measuring $3.2 \mathrm{~mm}(Z$-score +2.5 for body surface area).

strongly supports this conclusion. Consistent with the literature, the identified coronary abnormalities have regressed with time. Three yr after transplant, the right and left main coronary arteries are normal in size. The aneurysm of the LAD persists, although it is smaller than it was on angiography one yr prior.

The clinical implications of these findings are unclear, but there is evidence to suggest that abnormalities of coronary artery morphology and function persist following Kawasaki disease even after any echocardiographic and angiographic changes have resolved. Several groups have documented persistent intimal thickening following normalization of coronary artery dimensions $(4,5)$, and there are multiple reports of coronary endothelial dysfunction in the same

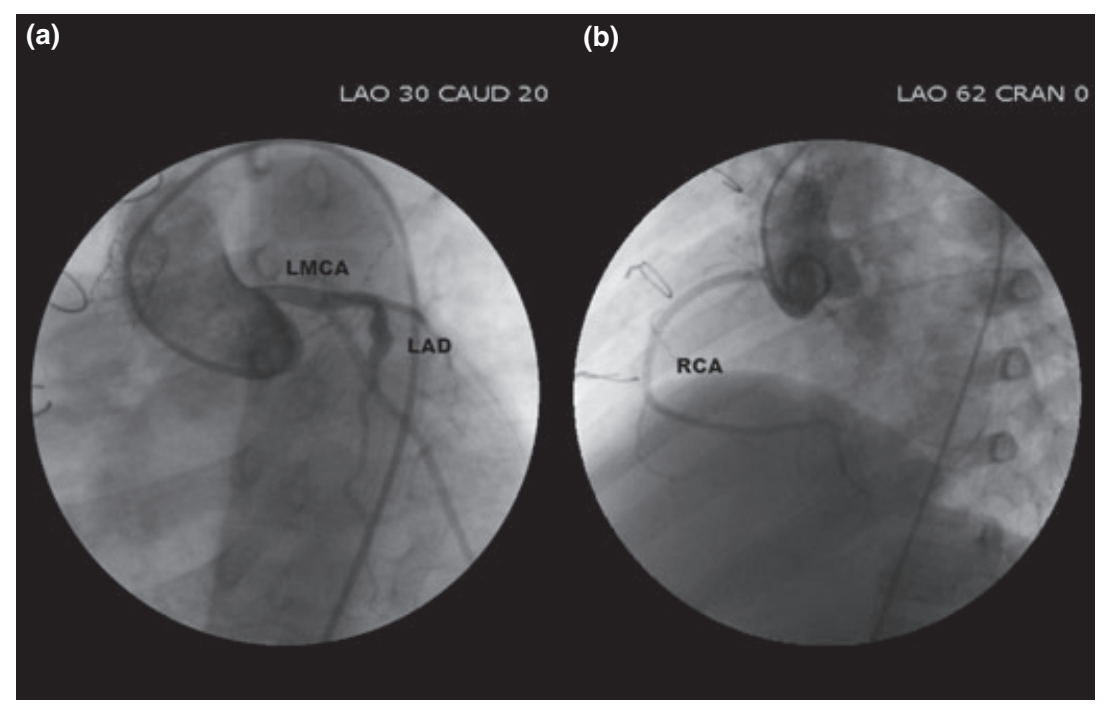

Fig. 1. (a) Initial screening coronary angiography two yr after transplant revealed a dilated LMCA measuring 4.0 mm $(Z$-score +3.9 for body surface area, BSA $)$ and a fusiform aneurysm of the proximal LAD measuring $3.8 \mathrm{~mm}(Z$-score +6.8 for BSA). (b) The proximal RCA was dilated as well, measuring $3.6 \mathrm{~mm}$ ( $Z$-score +4.9 for BSA). 
setting (6-8). These findings have led to speculation that patients with coronary involvement related to Kawasaki disease, even those in whom all visible lesions have regressed, may be at risk of premature atherosclerotic coronary artery disease. Given the link between coronary endothelial dysfunction and the development of TCAD (9), it is worrisome that the effects of Kawasaki disease on the graft in this case may also increase the risk of early TCAD.

Based upon the AHA guidelines for Kawasaki disease, this patient would fall in risk level III (small-to-medium coronary artery aneurysm). Recommended follow-up for patients in this risk class includes low-dose aspirin therapy and biannual non-invasive screening for coronary ischemia (stress test or myocardial perfusion scan) (2). Given the unknown long-term consequences of Kawasaki disease on coronary arteries in a transplanted heart, we have opted to monitor the patient with yearly coronary angiography for the time being, in addition to standard low-dose aspirin therapy. We will also consider early initiation of statin therapy and transition to sirolimus for its potential coronary protective effects (10).

\section{Conclusion}

To our knowledge, this is the first reported case of apparent undiagnosed Kawasaki disease in a cardiac organ donor manifesting with coronary artery ectasia and aneurysm formation in the organ recipient. Consistent with the natural history of Kawasaki disease, the right and left coronary artery ectasia has resolved three yr after transplant, but a small aneurysm of the LAD persists. Based on numerous reports of persistent coronary artery intimal thickening and endothelial dysfunction in the aftermath of Kawasaki disease, we believe that this patient may be at increased risk of developing early TCAD. Continued close monitoring is warranted, along with standard medical coronary protective therapy.

\section{Authors' contributions}

Joshua Friedland-Little and Ranjit Aiyagari were involved in case report conception, data collection, data interpretation, manuscript preparation and revision, and final approval. Robert Gajarski and Kurt Schumacher were involved in case report conception, data interpretation, manuscript preparation and revision, and final approval.

\section{References}

1. Kawasaki T. Acute febrile mucocutaneous syndrome with lymphoid involvement with specific desquamation of the fingers and toes in children. Arerugi 1967: 16: 178-222.

2. Newburger JW, Takahashi M, Gerber MA, et al. Diagnosis, treatment, and long-term management of Kawasaki disease: a statement for health professionals from the Committee on Rheumatic Fever, Endocarditis, and Kawasaki Disease, Council on Cardiovascular Disease in the Young, American Heart Association. Pediatrics 2004: 114: 1708-1733.

3. Kato H, Sugimura T, Akagi T, et al. Long-term consequences of Kawasaki disease. A 10- to 21-year follow-up study of 594 patients. Circulation 1996: 94: 1379-1385.

4. Tsuda E, Kamiya T, Kimura K, Ono Y, Echigo S. Coronary artery dilatation exceeding $4.0 \mathrm{~mm}$ during acute Kawasaki disease predicts a high probability of subsequent late intima-medial thickening. Pediatr Cardiol 2002: 23: 9-14.

5. Iemura M, Ishi M, Sugimura T, Akagi T, Kato H. Long term consequences of regressed coronary aneurysms after Kawasaki disease: Vascular wall morphology and function. Heart 2000: 83: 307-311

6. Furuyama H, Odagawa Y, Katoh $\mathrm{C}$, et al. Altered myocardial flow reserve and endothelial function late after Kawasaki disease. J Pediatr 2003: 142: 149-154.

7. Matsumura K, Okuda Y, Ito T, Hirano T, Takeda K, YAMAGUCHI N. Coronary angiography of Kawasaki disease with the coronary vasodilator dipyridamole: assessment of distensibility of affected coronary arterial wall. Angiology 1988: 39: 141-147.

8. Sugimura T, Kato H, Inoue O, Takagi J, Fukuda T, Sato N. Vasodilatory response of the coronary arteries after Kawasaki disease: Evaluation by intracoronary injection of isosorbide dinitrate. J Pediatr 1992: 121: 684-688.

9. Zimmer RJ, Lee MS. Transplant coronary artery disease. JACC Cardiovasc Interv 2010: 3: 367-377.

10. Keogh A, Richardson M, Ruygrok P, et al. Sirolimus in de novo heart transplant recipients reduces acute rejection and prevents coronary artery disease at 2 years: A randomized clinical trial. Circulation 2004: 110: 2694-2700. 